\title{
QSONG: Supermassive Black Holes in Quasars at World's End
}

\author{
Myungshin $\operatorname{Im}^{1}$ and the QSONG Team \\ ${ }^{1}$ Center for the Exploration of the Origin of the Universe \\ Department of Physics \& Astronomy, Seoul National University, \\ Seoul, Korea \\ Email: mim@astro.snu.ac.kr
}

\begin{abstract}
We present the initial results of the AKARI mission program QSONG (Quasar Spectroscopic Observation with NIR Grism). QSONG utilizes the unique AKARI capability of performing spectroscopic observations at $2.5-5 \mu \mathrm{m}$, with the aim of understanding the mass evolution of $\sim 200$ quasars at $3.4<z<6.5$ from their rest-frame optical spectra. The program also studies the rest-frame NIR spectra of 99 well-studied quasars at low redshift $(z<0.5)$ and 10 red quasars. With the high-redshift QSONG observations, we detect and resolve redshifted optical lines such as $\mathrm{H} \alpha$ for the first time at this redshift, allowing us to measure the supermassive black hole (SMBH) masses at high redshift using the well-calibrated optical mass estimators and provide an independent assessment of UV line-based mass measurements whose reliability has been controversial. Until the launch of JWST, AKARI will be the only facility in the world capable of studying the rest-frame optical spectra of high-redshift objects out to $z \sim 6$. Our initial QSONG result indicates a lack of very massive SMBHs at $z>5.5$, suggesting that we are possibly witnessing the cosmic growth history of the most massive SMBHs $\left(\sim 10^{10} M_{\odot}\right)$ in the early universe. Ultimately, an extensive study of the rest-frame optical spectra of high-redshift QSOs will help us understand the evolution and the growth of SMBHs in the early universe.
\end{abstract}

Keywords. early universe, galaxies: active, galaxies: high redshift, quasars: emission lines

\section{Introduction}

Supermassive black holes are those with masses in the range $\sim 10^{5}-10^{10} M_{\odot}$. They are found at the hearts of spheroidal components of massive galaxies, and are the power sources of AGN such as quasars. Studies of SMBHs in SDSS quasars from $z=0$ to $z=4.55$ show that there exist SMBHs as massive as $10^{10} M_{\odot}$ out to $z \sim 4.5$, and their mass function evolves significantly between $z=2$ through $z=4.5$ (Shen et al. 2008; Vestergaard et al. 2008). Luminous quasars are now identified out to $z \sim 6$, and these quasars at the highest redshifts are believed to harbor SMBHs with $M \sim 10^{9} M_{\odot}$ (Fan et al. 2003; Willott et al. 2009).

Growing such massive SMBHs so early in the universe, however, is not an easy task, requiring the continuous accretion of matter at the Eddington rate for about 1 Gyr or so. To enable the formation of very massive BHs more easily, theoretical models introduce massive $\mathrm{BH}$ seeds with $M \sim 10^{6} M_{\odot}$, or super-Eddington accretion (Volonteri \& Rees 2005; Yoo \& Miralda-Escude 2004; Di Matteo et al. 2008; Sijacki et al. 2009). These ingredients in theoretical models need to be checked by observations.

In this regard, SMBHs with the highest masses are interesting objects to study. Since they are the heaviest BHs, one can trace their growth history by simply following how the heaviest SMBHs emerged throughout the history of the universe. When active, they are the most luminous AGN, making them observable even at very high redshift. So, from the observational point of view, they are relatively easy to study in detail. 
Previous ground-based and space-based observations of distant, luminous quasars have covered most of their spectral energy distributions from X-ray to radio. However, the restframe optical spectra of high-redshift quasars are redshifted into the infrared, $2.5 \mu \mathrm{m}$ to $5 \mu \mathrm{m}$, which remains unexplored territory due to observational limitations of currently operating facilities. Yet this is a key wavelength range. The rest-frame optical region is well understood for low-redshift quasars and contains important diagnostic features such as the Balmer lines, [O III], [O II], and the Fe II blends.

Meanwhile, the $2.5-5.0 \mu \mathrm{m}$ wavelength range contains NIR hydrogen lines and PAH features at $\sim 3.3 \mu \mathrm{m}$ which have yet to be studied extensively in low-redshift quasars.

$A K A R I$, an infrared space telescope, now offers a NIR spectrograph that can obtain spectra of quasars at $2.5-5.0 \mu \mathrm{m}$. Thus, we are now conducting a spectroscopic study of quasars in the distant universe using $A K A R I$ to tackle the questions related to SMBH formation in the universe.

\section{QSONG}

The Quasar Spectroscopic Observation with NIR Grism (QSONG) program s a study of distant quasars using the unique spectroscopic capabilities of the $A K A R I$ space telescope (Murakami et al. 2007). The AKARI mission is led by JAXA, with the participation of ESA and Korea. QSONG is one of the mission programs of $A K A R I$ (PI: H.M. Lee).

A unique capability of $A K A R I$ is low-resolution spectroscopy in the wavelength range 2.5-5 $\mu \mathrm{m}$ (Onaka et al. 2007; Ohyama et al. 2007). AKARI is equipped with an NIR grism $\left(R \sim 130\right.$, or FWHM $\left.\sim 2300 \mathrm{~km} \mathrm{~s}^{-1}\right)$ and an NIR prism $(R \sim 20)$ that can disperse the incoming light into a $2.5-5 \mu \mathrm{m}$ spectrum. The spectrum can be taken without any slit mask, or with a small $1^{\prime} \times 1^{\prime}$ aperture. The low background in space makes the $A K A R I$ NIR spectrograph currently the most sensitive device in this wavelength range. Indeed, it is the only existing facility that can take the rest-frame optical spectra of faint distant quasars until the launch of JWST. Since typical quasar broad emission-line widths have FWHM $\sim 4000 \mathrm{~km} \mathrm{~s}^{-1}$, the NIR grism mode provides adequate spectral resolution to resolve such lines.

Taking advantage of this unique capability of $A K A R I$, QSONG conducts spectroscopic studies of quasars at low redshift (low redshift QSONG, or LQSONG), and at high redshift (high redshift QSONG, or HQSONG). Both programs are summarized below.

\subsection{HQSONG - QSONG at High Redshift}

In HQSONG, we study the rest-frame optical spectra of quasars at $3.4<z<6.4$. The scientific objectives of HQSONG are (i) to determine the masses of supermassive black holes by measuring $\mathrm{H} \alpha$ and $\mathrm{H} \beta$ line widths and the $L(5100)$ continuum, (ii) to test the correlation of $L(5100)$ vs. Balmer-line fluxes, (iii) to investigate the significance of the narrow-line regions through the detection of [O III $\lambda 5007$, (iv) to investigate the chemical enrichment history through the Fe complexes, and (v) to look for evidence of significant amounts of star-forming activity by measurement of the [O II] $\lambda 3727$ luminosity.

The emission lines such as $\mathrm{H} \alpha$ and $\mathrm{H} \beta$ are redshifted into the 2.5-5.0 $\mu \mathrm{m}$ wavelength window at redshifts $3<z<6.5$, where the ground-based spectroscopy of faint, distant quasars is nearly impossible. The Spitzer Space Telescope lacked spectroscopic capability in this wavelength range (Figure 1).

The HQSONG sample is comprised of 164 quasars at $3.4<z<6.5$, primarily from SDSS. BR and BRI quasars in the southern sky are also included. The targets selected at redshifts $z<5.5$ have $z$-band magnitudes $z<19 \mathrm{AB}$ mag. For the higher-redshift $(z>5.5)$ targets, the magnitude $z$-band limit is $z<20 \mathrm{AB}$ mag. These limits are 
imposed in order to ensure that these sources are above the $A K A R I$ detection limit for spectroscopy, which is of order of a few hundred $\mu \mathrm{Jy}$. These magnitude limits pick up quasars with $L_{\mathrm{bol}}>10^{47} \mathrm{ergs} \mathrm{s}^{-1}$, and $M_{\mathrm{BH}}>10^{9} M_{\odot}$. During the $A K A R I$ warm mission, the detection limit increased from $500 \mu \mathrm{Jy}$ to $1 \mathrm{mJy}$. With a newly approved mission program, QSONG2, we are now conducting AKARI NIR spectroscopy of 33 additional distant quasars at $3.4<z<6.0$.

\section{2. $L Q S O N G-Q S O N G$ at Low Redshift}

LQSONG aims (i) to examine the characteristics of hydrogen lines at the poorly understood rest-frame wavelength region of $2.5-5 \mu \mathrm{m}$ and constrain the physical conditions of AGN line-emitting regions, and (ii) to establish diagnostics to study the SMBHs even in dust-reddened systems, e.g., by using NIR hydrogen lines as mass estimators. Establishing a mass estimator using NIR hydrogen lines is important, especially for quasars that are dust-reddened. In dust-reddened quasars, the common single-epoch mass estimators, such as those using Balmer lines or UV metal lines, are subject to great uncertainties in the amount of dust extinction. On the other hand, NIR hydrogen lines are much less affected by dust extinction, making them more useful mass estimators. To achieve these goals, we target bright, low-redshift QSOs with the $A K A R I$ NIR grism. Figure 1 shows the redshift versus the observed wavelength of key NIR hydrogen lines. With the $A K A R I$ grism, we will be able to sample $\operatorname{Br} \alpha$ at $z<0.2, \operatorname{Br} \beta$ at $z<0.8$, and $\operatorname{P} \alpha$ at $0.5<z<0.8$

The LQSONG sample is comprised mostly of 29 low-redshift quasars studied with reverberation mapping (Kaspi et al. 2000; Peterson et al. 2004), and 26 PG quasars at $z<0.5$ (Vestergaard \& Peterson 2006). LQSONG also includes four newly discovered

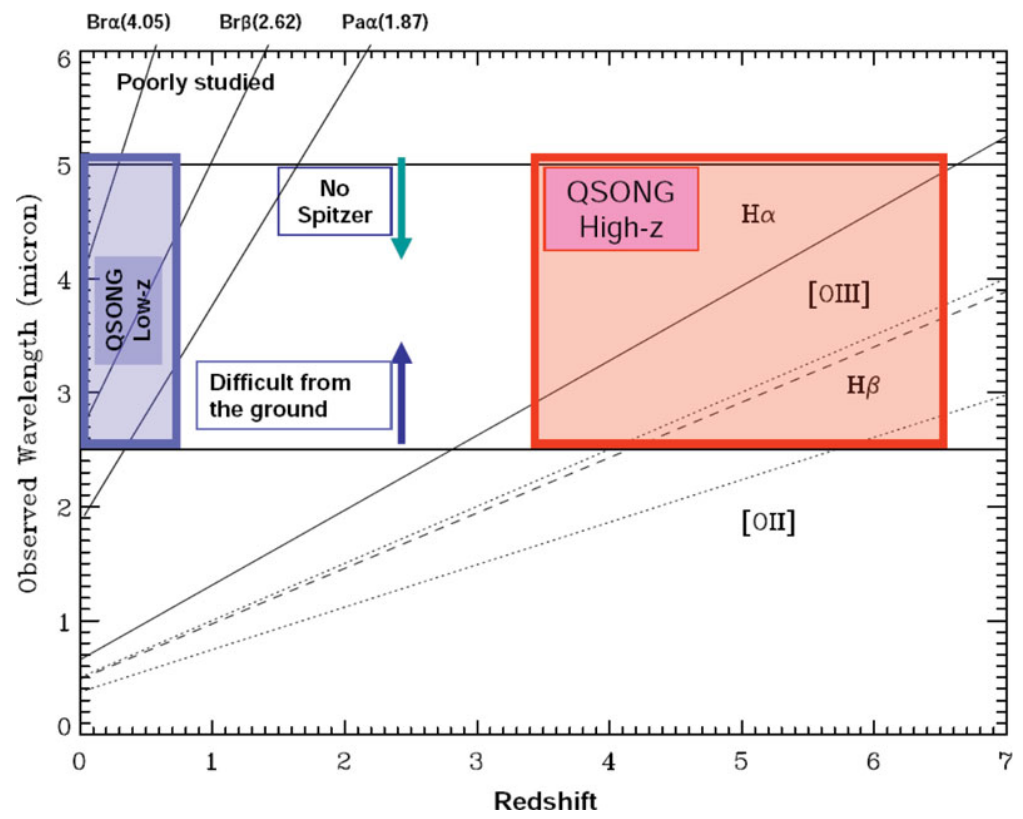

Figure 1. The observed wavelengths of key optical lines for AGN diagnostics as a function of redshift. The strongest Balmer line, $\mathrm{H} \alpha$, redshifts into the $2.5-5 \mu \mathrm{m}$ band at $3<z<6.5$, prompting a need for spectroscopy in this wavelength range, which is possible only with $A K A R I$. Our program, QSONG, targets this redshift and wavelength region as marked with a box on the right. $A K A R I$ can also study $\operatorname{Br} \alpha$ and $\operatorname{Br} \beta$ lines in well-studied, low-redshift AGNs. Such a study is also being carried out by QSONG (box on left). 
bright quasars from the Seoul National University Bright Quasar Survey (SNUQSO; Lee et al. 2008; Im et al. 2007), and 10 red quasars from Glikman et al. (2006). QSONG2 is now underway and will obtain NIR grism spectra of 33 additional PG quasars at $z<0.5$.

\section{Preliminary Results}

\section{1. $H Q S O N G$}

The data have been taken for all QSONG phase3a targets and the HZQSO open time program (PI: M. Im). Here, we show preliminary results from the HZQSO open time program which was carried out as a pilot QSONG program.

In HZQSO, 14 quasars at $z>4.5$ have been observed. We detect the redshifted $\mathrm{H} \alpha$ emission line in 9 of these targets. An example of an $\mathrm{H} \alpha$ detection is shown in Figure 2 , for BR0006-6224 at $z=4.51$. From the $\mathrm{H} \alpha$ luminosities and velocity widths, we estimate black hole masses using the local relation of McGill et al. (2007; also see Greene $\&$ Ho 2006). We find that SMBHs with $M \sim 10^{10} M_{\odot}$ exist out to $z \sim 5$, and for a few $\times 10^{10} M_{\odot}$ out to $z \sim 6$. However, it appears that the most massive SMBHs with $M \sim 10^{10} M_{\odot}$ seem to disappear at $z \sim 6$, suggesting that we are witnessing the emergence and the rapid growth of the most massive black holes at $z \sim 6$. We plan to publish a careful analysis of these results in near future (Figure 3; Im et al. 2010, in preparation).

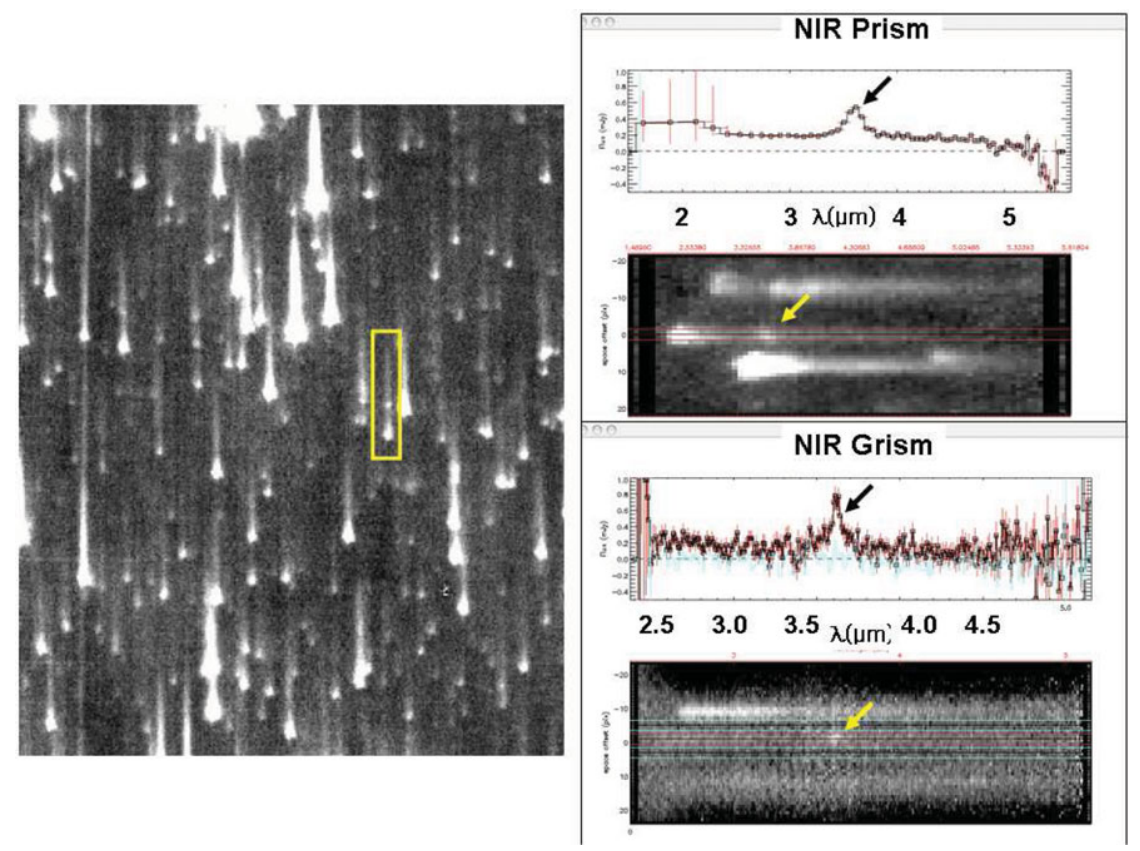

Figure 2. An example of an $A K A R I$ spectrum taken for the QSONG high-redshift targets. This figure shows the NIR spectra of BR0006-6224 at $z=4.51$. The left figure shows the slitless prism spectrum, where the boxed area indicates the prism spectrum of BR0006-0624. The top right shows the NIR prism spectrum, and the bottom right shows the NIR grism spectrum of the same object. The arrows indicate the location of the redshift $\mathrm{H} \alpha$ line at $3.61 \mu \mathrm{m}$. 


\section{2. $L Q S O N G$}

Like HQSONG, all the data have been taken up to $A K A R I$ Phase 3-I, and new data for 33 PG quasars are being taken for the AKARI Phase 3-II observations as a part of the QSONG mission program. The data taken up through Phase 3-I are being reduced now, with a sample spectrum of NGC 4151 shown in Figure 4. With these spectra, we expect to derive the $\mathrm{BH}$ mass estimator using Brackett lines and also apply a BH estimator from Paschen lines (Kim \& Im 2010, in preparation) to red AGN to derive their masses.

\section{Summary}

QSONG will obtain the rest-frame optical spectra of 200 quasars at $z>3.4$ (HQSONG program), and 2.5-5.0 $\mu \mathrm{m}$ spectra of about 100 nearby AGN (LQSONG program). We detect and resolve the redshifted $\mathrm{H} \alpha$ line in quasars at $z>4.5$ out to $z=6.22$ for the first time, enabling us to measure SMBH masses using the well-calibrated Balmer-line method. A preliminary result shows that $10^{10} M_{\odot}$ quasars exist out to $z=5.5$, but at $z=6$, they seem to disappear. The most massive SMBHs appear to be still growing at $z \sim 6$. The LQSONG program is also providing a new look at low-redshift AGN by capturing their $2.5-5 \mu \mathrm{m}$ spectra. We expect to provide useful AGN diagnostics from these spectra.

\section{Acknowledgements}

I thank $A K A R I$ QSONG members, especially Y. Ohyama, H. Jun, D. Kim, M. Kim, J. Woo, X. Fan, and H. M. Lee, for their contributions. This work was supported by the Korea Science and Engineering Foundation (KOSEF) grant No. 2009-0063616, funded by the Korea government (MEST). This research is based on observations with $A K A R I$, a JAXA project with the participation of ESA.

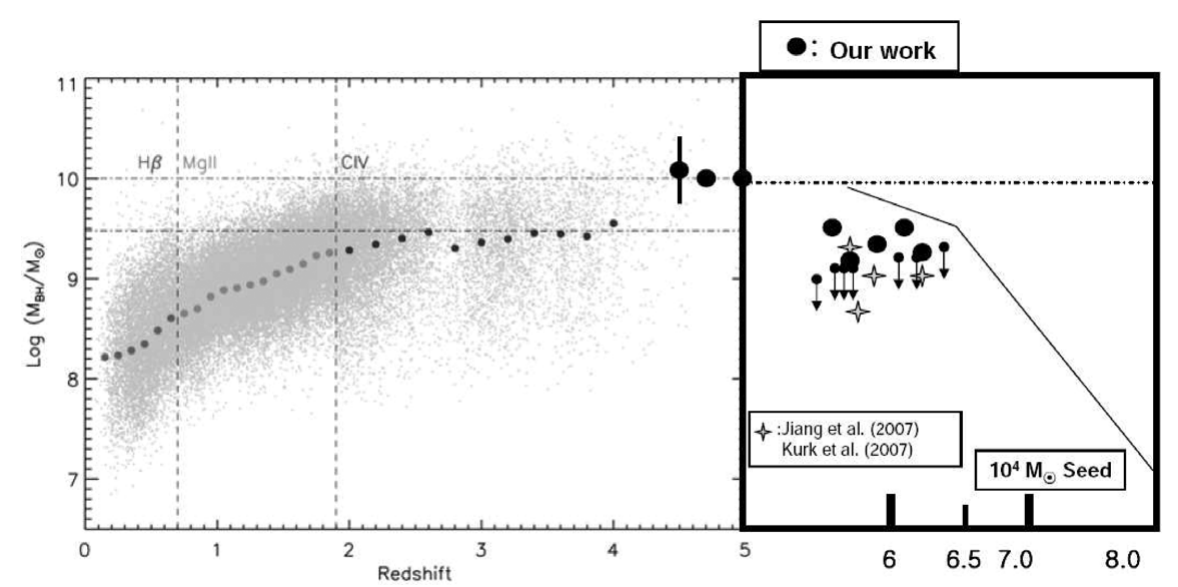

Figure 3. Redshift versus SMBH mass of quasars. The left figure is taken from Shen et al. (2008), showing the relation for SDSS quasars. Note the existence of $10^{10} M_{\odot}$ SMBHs out to $z \sim 4.5$. Our QSONG points are marked with black filled circles. Small circles with arrows indicate the upper limits. Note, also, Mg II-based measurements from the ground (stars). The solid line indicates possible growth of SMBHs before $z=6.5$. 


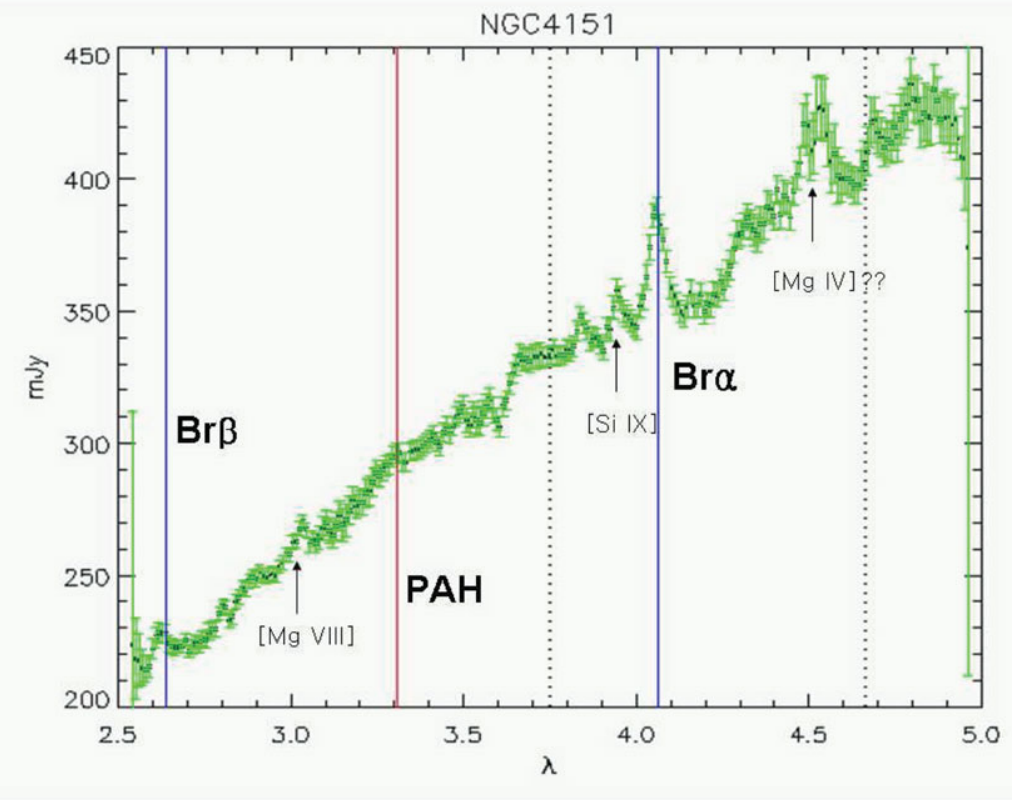

Figure 4. The AKARI spectrum of NGC 4151. The spectrum shows many key emission lines, with the most notable of these being $\operatorname{Br} \alpha$ and $\operatorname{Br} \beta$. Several more high-ionization emission lines are visible as well.

\section{References}

Di Matteo, T., et al. 2008, ApJ, 676, 33

Fan, X., et al. 2003, AJ, 125, 1649

Glikman, E., et al. 2007, ApJ, 667, 673

Greene, J. E. \& Ho, L. C. 2005, ApJ, 630, 122

Im, M., et al. 2007, ApJ, 664, 64

Jiang, L., et al. 2007, AJ, 134, 1150

Kaspi, S., et al. 2000, ApJ, 533, 631

Kurk, J., et al. 2007, ApJ, 669, 32

Lee, I., et al. 2008, ApJS, 175, 116

McGill, K., Woo, J., Treu, T., \& Malkan, M. A. 2008, ApJ, 673, 703

Murakami, H., et al. 2007, PASJ, 59, S369

Ohyama, Y., et al. 2007, PASJ, 59, S411

Onaka, T., et al. 2007, PASJ, 59, S401

Peterson, B. M., et al. 2004, ApJ, 613, 682

Shen, Y., et al. 2008, ApJ, 680, 169

Sijacki, D., Springel,V., \& Haehnelt, M. G. 2009, MNRAS, 400, 100

Vestergaard, M., et al. 2008, ApJ, 674, L1

Vestergaard, M. \& Peterson, B. M. 2006, ApJ, 641, 689

Volonteri, M. \& Rees, M. J. 2005, ApJ, 633, 624

Willott, C., et al. 2009, AJ, 137, 3541

Yoo, J. \& Miralda-Escude, J. 2004, ApJ, 614, L25 